\title{
SANTA CRUZ DE TENERIFE, 1936-1972. UN ESTUDIO DE SU HACIENDA MUNICIPAL DURANTE EL FRANQUISMO*
}

\author{
María del Pino Ojeda Cabrera \\ Ruymán Hernández Pacheco
}

\section{RESUMEN}

Este artículo estudia la hacienda municipal de Santa Cruz de Tenerife en un período crucial de su historia. Tiene como base documental las liquidaciones presupuestarias y se articula en dos apartados. El primero examina los ingresos, en los que desempeńa un papel esencial la denominada la Carta Económica Municipal, una fuente de financiación única y singular aportada por el Cabildo Insular de Tenerife. El segundo apartado estudia el gasto, que, al igual que el ingreso, experimentó un fuerte crecimiento durante la década de 1960, coincidiendo con la nueva inserción de la economía isleńa en el escenario internacional.

Palabras clave: hacienda municipal, dictadura de Franco, Santa Cruz de Tenerife, Carta Económica Municipal.

\section{SANTA CRUZ DE TENERIFE, 1936-1972. A STUDY OF ITS MUNICIPAL TREASURY DURING THE FRANCOISM}

\section{Abstract}

This article studies the municipal treasury of Santa Cruz de Tenerife at a crucial period in its history. It has as a base the budgetary settlements and is divided into two sections. The first examines the income, where the so-called Carta Económica Municipal plays an essential role, a unique source of funding provided by the Cabildo Insular de Tenerife. The second section studies the budgetary expenditure, which, like income, had a strong growth during the 1960s, coinciding with the new insertion of the island economy in the international scenario.

Keywords: Municipal treasury, Dictatorship of Franco, Santa Cruz de Tenerife, Carta Económica Municipal. 


\section{INTRODUCCIÓN}

La Guerra Civil y la posterior política autárquica incrementaron los niveles de miseria en una economía insular cuya principal actividad productiva dependía estrechamente del mercado internacional. La principal función del municipio de Santa Cruz de Tenerife, la urbana-portuaria, perdió toda vitalidad y, en consecuencia, mermaron los ingresos de su hacienda. Una hacienda que debió ahora adaptarse a una política fiscal que retornó a los viejos modelos, pues su principal fuente de financiación fueron los consumos. Ocurrió entonces lo que Barciela califica de injusticia fiscal; el gasto se vinculó con los sectores adeptos al nuevo régimen, mientras las necesidades sociales ocuparon los últimos escalones presupuestarios. Además, se produjo un acusado y creciente fraude fiscal, de modo que asistimos a una de las peores etapas de la historia tributaria del país'.

La Carta Económica Municipal fue la aportación financiera del Cabildo Insular de Tenerife a las arcas municipales y alivió sus penurias durante los años finales de la década de 1950, cuando esta hacienda municipal inició una etapa de febril crecimiento, coincidiendo con la apertura exterior de la economía isleńa. Ahora, las novedades vinieron a instancias del ascendente protagonismo del sector público, de la presencia de capital peninsular y del fomento del turismo. Los resultados se midieron en términos de crecimiento y de bienestar relativo, aunque ello no estuvo exento de incertidumbres a medio y largo plazo. La expansión urbana de Santa Cruz de Tenerife adquirió entonces una nueva dimensión, pues la sustancial mejora de la economía incrementó la renta de la población y, por ende, los ingresos y los gastos municipales.

Este nuevo marco de fiscalidad municipal se vio favorecido por el Plan de Estabilización y Liberalización de 1959, que marcó el inicio de la alineación de España en el escenario internacional ${ }^{2}$, y se vio además acompañado por reformas fiscales a nivel local (1962, 1964 y 1966) con el objetivo de simplificar las figuras tributarias y dotar de autonomía financiera a las corporaciones locales. Se trataba, ahora, de atender el creciente gasto de las administraciones públicas. Además, los sucesivos Planes de Desarrollo favorecieron también el nuevo escenario, al invertir en educación, sanidad y en transferencias a la Seguridad Social ${ }^{3}$.

* AMSCT = Archivo Municipal de Santa Cruz de Tenerife.

1 Barciela, Carlos: «Guerra civil y primer franquismo (1936-1959)», en Comín, Francisco, Hernández, Mauro y Llopis, Enrique (eds.): Historia económica de España siglos X y XX, Barcelona, 2005, pp. 349-351. Comín, Francisco: Historia de la Hacienda pública, II (1808-1995), Barcelona, 1996; Díaz, Daniel: «Fraude y amnistías fiscales en la España contemporánea, 1940-1990», Hacienda Pública Española, 1 (1994), pp. 389-402.

2 Serrano, José María y Pardos, Eva: «Los años de crecimiento del franquismo (19591975)", en Comín, Francisco, Hernández, Mauro y Llopis, Enrique (eds.): Historia Económica de España, Siglos X-XX, Barcelona, 2005, pp. 370-371.

${ }_{3}$ Fueron tres los Planes de Desarrollo que se aplicaron: el primero, aprobado en 1963 y aplicado desde 1964 hasta 1967, el segundo de 1968 a 1971 y el tercero de 1972 a 1975, aunque interrumpido por la crisis del petróleo de 1973. 
Las fuentes de este estudio son las liquidaciones presupuestarias ${ }^{4}$; de 1936 a 1943 son las rendidas por el Alcalde Presidente del municipio, y para los años 1944-1958 la Cuenta que rinde el depositario de los fondos municipales, que recoge la recaudación de los ingresos y los gastos invertidos en el transcurso del ejercicio presupuestario ${ }^{5}$.

Las liquidaciones de 1959 a 1962 se han obtenido de los libros diarios de ingresos y gastos, y las de los años de 1963 a 1971 de los Anuarios Estadísticos del Instituto Nacional de Estadística (INE), dada la ausencia de esta información en el AMSCT. No obstante, los Anuarios proporcionan únicamente los valores totales de cada capítulo, por lo que hemos recurrido a fuentes complementarias como los presupuestos ordinarios, aunque no hemos localizado algunos ańos (1971-1972). Por último, el presupuesto liquidado de 1972 se construyó mediante la Cuenta de Caudales del Depositario ${ }^{6}$.

El trabajo consta de dos partes. La primera estudia los ingresos presupuestarios liquidados del municipio y destaca el protagonismo de la Carta Económica Municipal en el sistema de financiación local. La segunda examina los gastos liquidados teniendo en cuenta el gasto por habitante, las deudas y las inversiones municipales.

\section{LOS INGRESOS LIQUIDADOS}

Desde el inicio de la Guerra Civil hasta 1954, su tendencia fue de contracción y claro estancamiento (cf. figura 1). A partir de entonces comenzó una tendencia alcista debido, sobre todo, a la aplicación del Decreto de 1955, que creó nuevos recursos fiscales con el fin de dotar de autonomía financiera a las corporaciones locales 7 .

Esta tendencia adquirió mayor relevancia en la década de 1960, cuando los ingresos se multiplicaron por cinco. Cabe, no obstante, observar dos intervalos diferenciados. El primero, de 1959 a 1963, respondió a una dinámica continuista

4 AMSCT.

${ }^{5}$ La periodicidad de esta cuenta es trimestral, de modo que la serie anual es la suma de los cuatro trimestres del ejercicio presupuestario. Por su parte, la temporalidad basada en el año natural o civil era la norma establecida en el período analizado por la Real Orden de 24 de junio de 1926. Véase RodríGuez, Eduardo: «Expansión y crisis de la hacienda municipal de Sevilla, 1924-1936», en GonzÁlez, Luis y Matés, Juan Manuel (coords.): La modernización económica de los ayuntamientos: servicios públicos, finanzas y gobiernos municipales, Jaén, 2008, p. 51. Esta norma quedó consolidada por la Ley de 17 de julio de 1945 de Bases de Régimen Local (base 65).

${ }^{6}$ Solamente hemos podido localizar el cuarto trimestre, pero este documento recoge las liquidaciones trimestrales anteriores, de modo que podemos conocer la liquidación anual.

7 Se regularon los arbitrios municipales a partir de una tributación propia y se suprimieron los recargos sobre las contribuciones urbanas y rústicas, que se vieron sustituidas por los arbitrios sobre la riqueza urbana y rústica. Paradójicamente, se aumentaron los recargos sobre la contribución industrial y comercial y se crearon otros dentro de la tributación provincial. Véase COMÍN, ob. cit., pp. 230-231. 
Figura 1. Evolución de los ingresos liquidados y del ingreso liquidado por habitante, 1936-1972 (en miles de pesetas constantes de 2000).

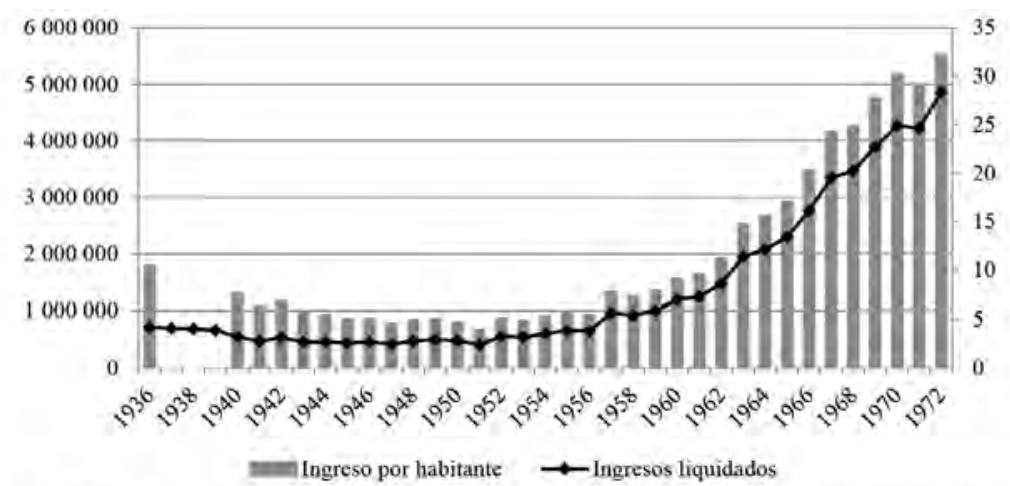

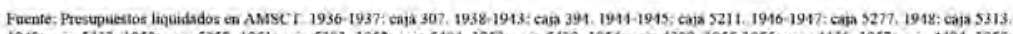
1949: caja 5333. 1950: caja 5358. 1951: caje 5383. 1952: caja 5404. 1953: cajn 5439. 1954: caja 4399. 1955-1956; caja 1436. 1957: caja 4494. 1958:

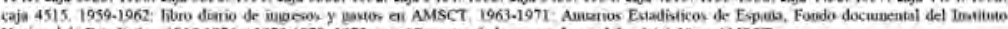
Nacional de Estadistica. 1966-1974 y 1978-1979. 1972 : caja "Carpetas de la coenta de catushales 4.1.1.1" en AMSCT.

por efecto de la política estabilizadora que había iniciado el régimen ${ }^{8}$; en concreto, aludimos a la reforma tributaria de Mariano Rubio (1957), cuyo objetivo fue ampliar los ingresos y contener el gasto con el fin de incrementar el ahorro del sector público', y al Plan de Estabilización de 1959. En el segundo (1964-1972), el incremento de los ingresos fue aún más significativo; se multiplicaron por 2,3, pasando de 2076 millones de pesetas en 1964 a más de 4858 millones en 1972. Ello debe relacionarse con el despegue del sector de la construcción y de los servicios vinculados a los inicios de la expansión del turismo ${ }^{10}$.

En cuanto al ingreso por habitante, la ratio alcanzada durante la II República se truncó con el estallido de la Guerra Civil (cf. figura 1). La hacienda santacrucera sufrió entonces una profunda depresión, que persistió durante todo el período autárquico. Si antes de 1936 el ingreso por habitante alcanzaba casi 10500 pesetas $^{11}$, se contrajo luego hasta el umbral de las 3800 pesetas al comenzar la década de 1950,

8 Ojeda, María del Pino y Hernández Pacheco, Ruymán: «La hacienda de Santa Cruz de Tenerife (1936-1958)", Documentos de trabajo de la Asociación Española de Historia Económica, 1702 (2017), pp. 13-14.

9 Véase el Plan de Desarrollo Económico y Social del período 1964-1967.

${ }^{10}$ Una variable que contribuye a argumentar el proceso de expansión urbana de estos años es el número de licencias comerciales concedidas en estos años, que en cifras brutas pasaron de 1703 en 1963 a 2994 en 1970. La mayor participación se dio en la rama vinculada a la alimentación, mientras que la de menor participación es la de energía eléctrica y mecánica, gas de ciudad y agua. En el resto de ramas se repartieron de forma más o menos homogénea. Con todo, cabe señalar que, en conjunto, el sector de la alimentación absorbió en torno al 50\% de las licencias concedidas. Véase Anuarios del Mercado Español (varios años).

${ }^{11}$ Una tendencia que venía marcada desde la etapa de la II República. Véase OJEDA, María del Pino: La Hacienda Municipal de Santa Cruz de Tenerife (1800-1936) (tesis doctoral inédita), Universidad de La Laguna, 2016. 
cuando presentó su nivel más bajo. La recuperación, aunque moderada, ocurrió en los dos últimos años de esta década, pero no volvieron a obtenerse los valores logrados antes de la guerra.

Se trató de una conquista alcanzada en los años sesenta. Entre 1959 y 1963 el ingreso per cápita se multiplicó por 1,9, al pasar de 7800 a 14650 pesetas, y a ello contribuyó un menor crecimiento poblacional del municipio ${ }^{12}$. Pero entre 1964 y 1972, este indicador se multiplicó en la misma proporción que en el subperíodo anterior, a pesar de que las cantidades liquidadas en concepto de ingresos aumentaron en mayor cuantía.

En síntesis, de 1959 a 1972, el ingreso por habitante quedó multiplicado por cuatro, demostrando que fue el volumen de ingresos liquidados lo que contribuyó al aumento de este indicador y no la conducta de la población, que se mantuvo relativamente estable. Esto lo corrobora la tasa anual de crecimiento acumulativo: la del ingreso fue del 11,5\% y la de la población del 1,3\%.

\subsection{LOS INGRESOS FISCALES Y LA PRESIÓN FISCAL}

Todo lo dicho queda patente si se examinan los capítulos de los presupuestos liquidados de ingresos que ilustran el cómputo de la presión fiscal (cf. tabla 1), teniendo en cuenta, además, el marco legislativo impuesto por la Dictadura ${ }^{13}$. Esta nueva perspectiva analítica permite comprobar que no hubo crecimiento de la presión fiscal durante el primer franquismo; cabe incluso aludir a su caída, alcanzando un mínimo de 2500 pesetas en 1951.

\begin{tabular}{|c|c|c|c|c|c|c|c|}
\hline AÑos & $\begin{array}{l}\text { INGRESOS } \\
\text { FISCALES }\end{array}$ & $\begin{array}{c}\text { Presión } \\
\text { FISCAL }\end{array}$ & $\begin{array}{c}\text { Presión FisCAL } \\
\text { REAL (\%) }\end{array}$ & AÑos & $\begin{array}{l}\text { INGRESOS } \\
\text { FISCALES }\end{array}$ & $\begin{array}{c}\text { Presión } \\
\text { FISCAL }\end{array}$ & $\begin{array}{l}\text { PRESión Fis- } \\
\text { CAL REAL (\%) }\end{array}$ \\
\hline 1936 & 355665,7 & 5,2 & 49,7 & 1955 & 482375,0 & 4,1 & 73,3 \\
\hline 1937 & 311 146,3 & & & 1956 & 467408,3 & 3,9 & 71,6 \\
\hline 1938 & 347914,0 & & & 1957 & 786957,5 & 6,3 & 81,8 \\
\hline 1939 & 332248,8 & & & 1958 & 780560,5 & 6,1 & 84,8 \\
\hline 1940 & 300551,3 & 4,2 & 55,1 & 1959 & 831034,2 & 6,5 & 83,2 \\
\hline
\end{tabular}

12 La población de hecho Santa Cruz pasó de 128235 habitantes en 1959 a 133078 en 1963 , lo que supone una tasa anual de crecimiento acumulativo del $0,93 \%$, mientras que, entre ambas fechas, la ratio de ingreso per cápita creció en una tasa del 17,2\%.

13 Para calcular los ingresos fiscales de 1936 a 1958, se ha utilizado el capítulo 7, de contribuciones especiales; el capítulo 8, de derechos y tasas; el capítulo 9, de cuotas, recargos y participaciones en tributos nacionales; y el capítulo 10, de imposición municipal, descartando los que se imponen con fines no fiscales. Véase Rovira, Alberto: «Los llamados arbitrios con fines no fiscales», Revista de Estudios de la Vida Local, 99 (1958), p. 365. 


\begin{tabular}{rrrlllll}
\hline 1941 & 299873,8 & 4,0 & 63,9 & 1960 & 956741,6 & 7,3 & 79,9 \\
\hline 1942 & 322517,4 & 4,1 & 60,5 & 1961 & 1020088,0 & 7,8 & 82,1 \\
\hline 1943 & 280344,9 & 3,4 & 61,4 & 1962 & 1118658,0 & 8,5 & 76,0 \\
\hline 1944 & 270129,0 & 3,2 & 59,1 & 1963 & 1316337,9 & 9,9 & 67,5 \\
\hline 1945 & 257946,0 & 2,9 & 59,5 & 1964 & 1428103,4 & 10,6 & 68,8 \\
\hline 1946 & 281101,0 & 3,1 & 61,7 & 1965 & 1626641,6 & 12,0 & 70,7 \\
\hline 1947 & 285261,3 & 3,0 & 68,4 & 1966 & 1865262,7 & 13,6 & 67,5 \\
\hline 1948 & 309527,5 & 3,2 & 65,4 & 1967 & 2187719,2 & 15,8 & 65,3 \\
\hline 1949 & 341613,2 & 3,4 & 68,4 & 1968 & 2267469,1 & 16,2 & 65,4 \\
\hline 1950 & 310823,0 & 3,0 & 65,3 & 1969 & 2662868,6 & 18,9 & 68,4 \\
\hline 1951 & 266506,2 & 2,5 & 65,6 & 1970 & 2791273,6 & 19,7 & 65,4 \\
\hline 1952 & 355786,9 & 3,3 & 64,5 & 1971 & 2948004,6 & 20,3 & 69,8 \\
\hline 1953 & 343350,8 & 3,1 & 63,7 & 1972 & 3091643,6 & 20,5 & 63,6 \\
\hline 1954 & 460418,9 & 4,0 & 76,9 & & & & \\
\hline
\end{tabular}

Fuente: ídem figura 1.

La recuperación de la ratio ocurre en los últimos años de la década de los cincuenta y su causalidad debemos buscarla en las modificaciones introducidas por la legislación de bases de Régimen Local, especialmente la referida a $1955^{14}$. En concreto, tuvo un efecto directo sobre el capítulo 10, de imposición municipal, sobre todo en los artículos referidos a los arbitrios sobre el consumo de bebidas espirituosas y alcoholes, cuyos ingresos a partir de dicha fecha superaron las 22000000 pesetas $^{15}$. También cabe citar el artículo alusivo a los arbitrios sobre carnes, volatería, caza menor, pescados y mariscos finos, que en dicho año ingresó en las arcas municipales cerca de 11675000 pesetas. Igualmente destacó el arbitrio sobre la riqueza urbana y las exacciones especiales o tradicionales ${ }^{16}$. El primero superó 59 millones pesetas en 1955 y 79 millones en 1958. Por su parte, los arbitrios especiales o tradicionales, con 88 millones en 1955, superaron los 359 millones en 1958. De ahí que fueran estos dos gravámenes los que contribuyeron a incrementar el volumen de ingresos liquidados, el ingreso liquidado por habitante y la presión fiscal en los años finales del primer franquismo.

Los ingresos fiscales del intervalo 1959-1972 se han calculado teniendo en cuenta el cambio en la estructura de los presupuestos impuesta en $1958^{17}$. En estos

14 Decreto de 24 de junio de 1955. Véase Ojeda y Hernández, ob. cit., pp. 6-8.

15 Véase apéndice de Ojeda y Hernández, ob. cit. Las cifras han sido deflactadas a pesetas constantes de 2000.

${ }^{16}$ Ley de 3 de diciembre de 1953 de Bases de Régimen Local, Base 10.

17 Se han considerado como ingresos fiscales las contribuciones especiales (capítulo 3, artículo 3), tasas por prestación de servicios y por aprovechamientos especiales (capítulo 3, artículos 1 y 2), impuestos directos (capítulo 1), impuestos indirectos (capítulo 2) y subvenciones y participaciones 
años la presión fiscal se triplicó, al pasar de 6500 a 20500 pesetas (cf. tabla 1). Este incremento exige examinar con cierto detalle este tramo, teniendo en cuenta la normativa fiscal, así como las estrategias de política económica plasmadas en los diferentes Planes de Desarrollo. En efecto, entre 1959 y 1963 la presión fiscal se multiplicó por 1,5, generando un incremento de 3400 pesetas, lo que debe relacionarse con la política estabilizadora del régimen. Pero observamos que en los últimos años su tendencia quedó moderada, especialmente a partir de 1969.

Interesaría ahora analizar la proporción que ocupa la presión fiscal con respecto al ingreso total por habitante, que hemos denominado presión fiscal real (cf. tabla 1). Los resultados son, a nuestro juicio, muy elocuentes. Así, en la primera etapa se observan los efectos de la autarquía, pues dicho indicador osciló entre el $50 \%$ y el $70 \%$, para incrementarse en más de 10 puntos porcentuales a partir del Plan de Estabilización y hasta 1961, y tocar techo en 1958 (85\%), en consonancia con las reformas y refundiciones legislativas introducidas. Desde 1962, coincidiendo con la dinámica económica aperturista (reformas tributarias, planes de desarrollo, etc.), comenzó su descenso de manera gradual, al disminuir 19,6 puntos porcentuales desde 1959 hasta 1972. Y de ello se deriva la relativa relajación de la presión fiscal soportada por la población; un hecho acorde con las estrategias político-económicas instauradas en este período.

Sin duda, los cambios legislativos tuvieron un claro objetivo: sanear o, al menos, minimizar los niveles de empobrecimiento presupuestario del municipio y alcanzar su autosuficiencia financiera. En este sentido, ya al inicio del período se comprueba cómo persistió la trayectoria emanada de la política preestabilizadora del régimen de finales de los años cincuenta ${ }^{18}$. De hecho, con el propósito de adaptarse a la nueva realidad económica, la primera reforma legislativa de esta etapa fue la Ley 85/1962, de 24 de diciembre, sobre reforma de Haciendas Municipales, que estableció la eliminación de la imposición sobre usos y consumos $^{19}$, obviando algunos epígrafes de la antigua tarifa quinta de dicha contribución, relativa a conceptos de lujo ${ }^{20}$.

en ingresos (capítulo 4). Para su cálculo entre 1963 y 1971, se han utilizado los datos de las series estadísticas del INE, donde solo constan los totales por capítulo, sin desglose de artículos que hagan referencia a su tipología fiscal o no fiscal. Por ello, en esos años se están incluyendo los arbitrios con fines no fiscales y los ingresos por concesiones administrativas; aunque se deduce que la representación de los primeros debió ser escasa a tenor de la trayectoria precedente en la que sí contamos con las liquidaciones detalladas. En relación con los segundos, no se cuenta con valor alguno para ninguno de los años estudiados; incluso, se ha recurrido a los presupuestos ordinarios, donde tampoco hay constancia de cantidad alguna presupuestada.

18 Ojeda y Hernández, ob. cit., pp. 7-9.

${ }^{19}$ Esta reforma, al suprimir casi en su totalidad los impuestos sobre el consumo, forzó a los ayuntamientos a sostenerse mayoritariamente con cargas directas. Un cambio que, dadas las características económico-fiscales de nuestro país, resultaba lento e ineficaz. Véase Rovira, Alberto: La hacienda municipal y sus problemas, Madrid, 1972, p. 95.

${ }^{20}$ En efecto, en Santa Cruz se ha constatado que se mantuvieron los epígrafes 19 y 27 de la misma. Incluían las consumiciones en hoteles y restaurantes de lujo, cabarets, bailes y similares. AMSCT. Presupuesto ordinario de Santa Cruz de Tenerife de 1966. 
El incremento de la presión fiscal también estuvo motivado por una nueva reforma de las haciendas locales en 1966, que centró su objetivo en la necesidad de aumentar los ingresos, y cuyo aspecto más notorio en este sentido fue la creación de un gravamen sobre la circulación de vehículos por la vía pública ${ }^{21}$. Además, la nueva reforma suprimió algunos recargos y refundió los que percibían los municipios sobre las cuotas que ingresaba el Tesoro de los impuestos directos, lo que supuso, en realidad, una simplificación administrativo-tributaria ${ }^{22}$. En virtud de ello, las varias contribuciones especiales establecidas en el Decreto refundido de 1955 se sustituyeron por una única contribución teniendo en cuenta el beneficio que generase, independientemente de su valor económico ${ }^{23}$.

\subsection{La estructura de los ingresos: municipio, Estado, Cabildo}

El análisis de la estructura de los ingresos municipales exige desglosar sus tres principales partidas: las aportaciones del Estado, que provienen de recargos y de la participación del municipio en los impuestos estatales; las del Cabildo Insular de Tenerife, que proceden de la Carta Económica Municipal y del arbitrio del tabaco; y, por último, los ingresos municipales propiamente dichos.

El Ayuntamiento se nutrió exclusivamente de ingresos estatales y municipales entre 1936 y 1956. Los primeros se integraban en el capítulo noveno de la estructura presupuestaria vigente y, específicamente, incluía la participación y recargos sobre las contribuciones de la hacienda estatal y los impuestos cedidos por el Estado al municipio. El resto de los ingresos eran específicamente municipales y de amplio espectro, abarcando desde tasas e impuestos hasta los extraordinarios e imprevistos, siendo la mayor parte de las exacciones de tipo indirecto. Estos ingresos oscilaron entre el $60 \%$ y el $75 \%$, mientras que los estatales representaron un promedio del $32 \%$ (cf. figura 2 ).

Los años de 1959 a 1972 constituyen un capítulo clave en la historia de la hacienda municipal de Santa Cruz de Tenerife. La Corporación debió asumir nuevos retos en su capítulo de gastos como consecuencia de la expansión urbano-portuaria de este período y, por consiguiente, debió desarrollar una política dirigida a buscar nuevas fuentes de financiación.

El modelo presupuestario fijado en la Ley de 1958 y las lagunas documentales impiden precisar las aportaciones del Estado durante los años de 1959 a 1962, mientras que las de los años de 1963-1972 se han obtenido del capítulo cuarto de Subvenciones y participaciones en ingresos, artículo primero del Estado. Durante estos años, la participación estatal en la estructura presupuestaria arroja un promedio del $15 \%$.

${ }^{21}$ Calvo, Rafael: «Medio siglo de hacienda municipal: del Estatuto al Proyecto de 1974», Hacienda Pública Española, 35 (1975), p. 171.

${ }^{22}$ Ley 48/1966.

${ }^{23}$ Calvo: ob. cit., pp. 167-168 
Figura 2. Estructura de los ingresos (municipio, Estado, Cabildo) (en \%), 1936-1972.

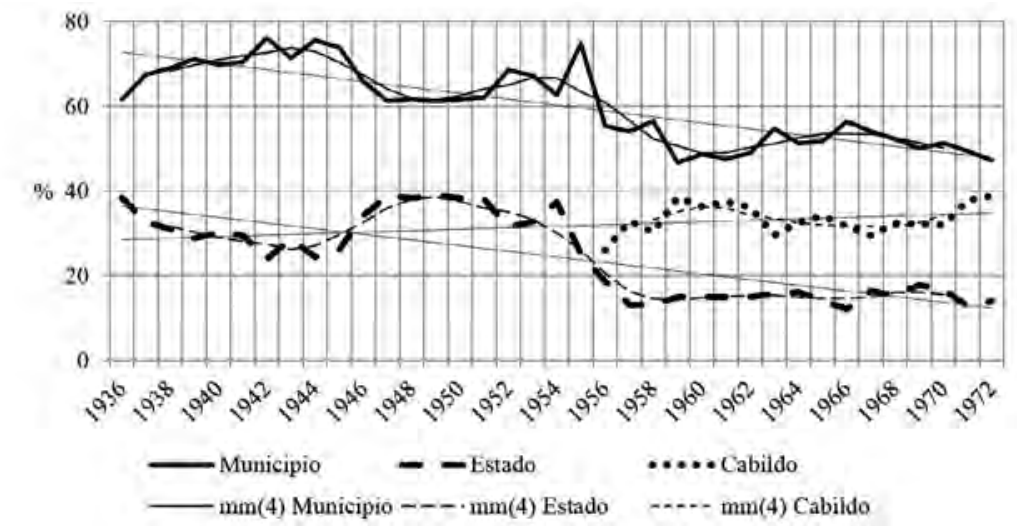

Fuente: Municipio y Estado: ídem figura 1. Cabildo: Boletín Oficial de la Provincia de Santa Cruz de Tenerife, 1959-1973.

La segunda vía de financiación, específicamente municipal, integra otra categoría de ingresos. En el período de 1959 a 1972 presentó una participación cuyo promedio fue del $50,7 \%$. Al contrastarla con los años del primer franquismo, su proporción se situó en un $66 \%$ de media, lo que se tradujo en una reducción del 15\%. Pero cabe observar que dicha merma se contraponía al dogma de la autosuficiencia financiera que la propia legislación venía planteando desde el siglo xix.

Finalmente, el Cabildo de Tenerife derivó parte de sus ingresos propios a la financiación de las haciendas municipales de la isla mediante la denominada Carta Económica Municipal y el arbitrio del tabaco ${ }^{24}$. La contribución del Cabildo a la

${ }^{24}$ El Cabildo Insular de Tenerife tenía autorizado el impuesto del 3\% ad-valorem sobre el arbitrio de importación y exportación de mercancías. La Corporación insular cedió el $1 \%$ de dicho impuesto a los municipios de la isla y autorizó un recargo de otro $1 \%$ por medio de dicha Carta, de modo que le correspondía un $2 \%$ al Cabildo y otro $2 \%$ a los ayuntamientos. Para su distribución se aplicaba un coeficiente de reparto que otorgaba el $60 \%$ del total de los ingresos obtenidos al Ayuntamiento de Santa Cruz de Tenerife. Los fondos de esta nueva fuente de imposición debían destinarse a obras y servicios de primer establecimiento (apertura y pavimentación de vías; alumbrado público; alcantarillado; alumbramiento, depósito y distribución de aguas; viviendas; casas consistoriales; mercados y mataderos; cementerios; casas escuelas; transportes; instrucción y cultura; fomento del turismo y otras dotaciones de la misma naturaleza). También podían utilizarse para pagar las operaciones de crédito que se hubieran contratado para realizar dichas obras y servicios. Véase Libro de Actas del Cabildo Insular de Tenerife de 1953, fol. 44'-48'. En cuanto el arbitrio del tabaco, el Ayuntamiento había logrado mediante Orden Ministerial de 20 de julio de 1934 la autorización de un arbitrio municipal sobre el consumo de tabaco elaborado. Desde 1937 el Cabildo Insular de Tenerife se encargó de gestionar este arbitrio, cuya recaudación repartía a todos los municipios de la isla teniendo en cuenta un coeficiente de reparto similar al de la Carta. Véase Miranda, Salvador: «Dos hitos en la hacienda de los Cabildos Insulares: la carta intermunicipal de 1941 y las funciones sociales desempeñadas con los arbitrios sobre la gasolina en 1927 y sobre el tabaco en 1937», Hacienda 
hacienda santacrucera fue de un 33,4\% durante la etapa de 1956-1972, lo que sin duda supuso una aportación económica considerable, pues llegaba justo cuando la participación del Estado se redujo a la mitad.

En síntesis, mientras la tendencia a largo plazo de las aportaciones del municipio y del Estado manifestaron un claro paralelismo descendente, a partir de 1956 se constata que la reducción del Estado fue sustituida por las aportaciones del Cabildo, con una evidente tendencia al alza debido al protagonismo creciente de la Carta Económica Municipal.

\section{LOS GASTOS LIQUIDADOS}

El gasto municipal marcó una trayectoria descendente hasta 1951, cuando se alcanzó el mínimo con 340898 millones de pesetas (cf. figura 3), lo que refleja la incapacidad inversora del régimen franquista por sus férreos condicionantes. Desde 1952 sus niveles comenzaron a mejorar tímidamente, siendo más destacada su recuperación a finales de la década de los cincuenta, al superarse los 900 millones de pesetas.

Desde del Plan de Estabilización y hasta 1972 se entró en una nueva dinámica; el volumen de los gastos pasó de cerca de 924 millones de pesetas y superó los 4375 millones en el último año, es decir, se multiplicaron por 4,7. Ahora bien, su trayectoria marcó dos intervalos diferenciados. En el primero, de 1959 a 1965, las cuantías liquidadas obedecen a una tendencia continuista ${ }^{25}$; en realidad, el mayor aumento en el gasto ocurrió a partir de 1966, coincidiendo con el impulso inversor del Plan Canarias ${ }^{26}$.

Por su parte, el gasto liquidado por habitante siguió la misma tendencia que el gasto total liquidado, pero los niveles del final de la II República no se alcanzaron hasta 1960. Durante los primeros años de la autarquía, la reducción llegó a marcar un mínimo de 3200 pesetas por habitante en 1951 debido al menor esfuerzo inversor efectuado por la Dictadura (cf. figura 3). Desde entonces, este indicador se incrementó lentamente, siendo más visible su recuperación en los últimos años de la década de los cincuenta; una mejoría solo equiparable a la inversión realizada antes de la Guerra Civil.

Canaria, 13 (2005), p. 78. YAnes, Julio Antonio: «El arbitrio municipal sobre el consumo de tabaco en Santa Cruz de Tenerife en la II República. Un ensayo fiscal insólito ante el fuerte endeudamiento de la dictadura de Primo de Rivera", Boletín Millares Carlo, 26 (2007), pp. 69-84. Aguiar, Carlos: La provincia de Santa Cruz de Tenerife entre dos dictaduras (1923-1945). Hambre y orden (tesis doctoral), Universitat de Barcelona, 2012, pp. 176-177; Libro de Actas del Cabildo Insular de Tenerife de 1937.

25 Ojeda y Hernández: ob. cit., pp. 21-23.

${ }^{26}$ González, Pedro: «Los planes de desarrollo en Canarias: el compromiso institucional del Cabildo Insular de Tenerife», en León, Aarón (coord.): El Franquismo en Canarias, La Laguna, 2014, pp. 468-469. 
Figura 3. Evolución de los gastos liquidados y del gasto liquidado por habitante, 1936-1972 (en miles de pesetas constantes de 2000).

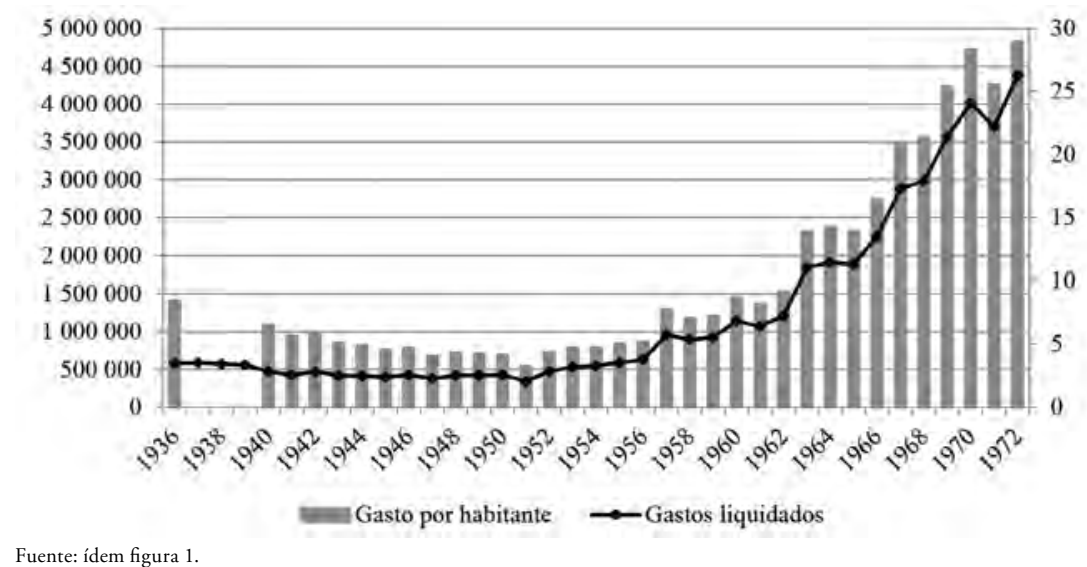

Desde 1959 hasta 1972, el incremento del gasto por habitante fue de tal magnitud que la tasa de variación alcanzó un 339,15\%. Ahora, la nueva conducta coincide con la etapa de apertura del régimen al exterior, donde la sinergia de los mercados internacionales también tuvo impacto en la modernización de las estructuras socioeconómicas. El gasto por habitante se multiplicó por cuatro, corroborando el incremento del gasto liquidado, al pasar de 7204 a 28944 pesetas.

\subsection{LAS DEUDAS}

En los capítulos de gastos, el de la deuda constituyó siempre uno de los más destacados de la Corporación municipal (cf. tabla 2). Así, en el primer franquismo, las operaciones de crédito municipal adquirieron relevancia al vincularse a los préstamos de entidades financieras, destacando los contraídos con el Banco de Crédito Local durante la década de $1920^{27}$. De hecho, el presupuesto ordinario de 1936 reconocía expresamente esta deuda histórica, al señalar que el Ayuntamiento continuaba pagando deuda interior como los «intereses ... al antiguo Hospital de San Carlos para la reconstrucción de la Recoba y el Teatro; ... del empréstito municipal de cinco

${ }^{27}$ Durante el franquismo, los estatutos del Banco de Crédito Local incluían la prohibición de prestar a los municipios cuantías cuyas amortizaciones anuales no superasen el $25 \%$ de los gastos de los presupuestos ordinarios. Además, se prohibía taxativamente la emisión de deuda a corporaciones con liquidaciones presupuestarias previas deficitarias. Véase Comín: ob. cit., p. 236. 
millones ${ }^{28} ;(. .$.$) del empréstito de diez millones { }^{29}$. Además, se siguieron abonando "gastos, intereses y amortización del empréstito de un millón doscientas cincuenta mil pesetas, para construcción del Palacio de Justicia $»^{30}$, a un interés del $6 \%$.

\begin{tabular}{|c|c|c|c|c|c|c|c|}
\hline \multicolumn{8}{|c|}{$\begin{array}{l}\text { TABLA 2. DEUDA LIQUIDADA Y DEUDA PRESUPUESTADA Y SU \% SOBRE } \\
\text { EL TOTAL DE GASTOS LIQUIDADOS Y PRESUPUESTADOS } \\
\text { (EN MILES DE PESETAS CONSTANTES DE 2000), 1936-1972 }\end{array}$} \\
\hline Años & $\begin{array}{c}\text { DEUDA } \\
\text { LIQUIDADA }\end{array}$ & $\%$ & AÑos & $\begin{array}{c}\text { DEUDA } \\
\text { LIQUIDADA }\end{array}$ & $\%$ & $\begin{array}{c}\text { DEUdA } \\
\text { PRESUPUESTADA }\end{array}$ & $\%$ \\
\hline 1936 & 98450,5 & 17,1 & 1955 & 35196,6 & 6,0 & & \\
\hline 1937 & 63415,4 & 10,8 & 1956 & 31945,4 & 5,1 & & \\
\hline 1938 & 138038,5 & 24,2 & 1957 & 32539,3 & 3,4 & & \\
\hline 1939 & 122416,5 & 22,0 & 1958 & 36449,7 & 4,1 & & \\
\hline 1940 & 99779,7 & 21,3 & 1959 & 40705,4 & 4,4 & 43777,0 & 4,5 \\
\hline 1941 & 83939,2 & 19,9 & 1960 & 34526,7 & 3,0 & 36978,9 & 3,9 \\
\hline 1942 & 85605,8 & 18,3 & 1961 & 26864,8 & 2,5 & 30287,4 & 2,9 \\
\hline 1943 & 59847,9 & 14,4 & 1962 & 27212,4 & 2,3 & 28782,5 & 2,4 \\
\hline 1944 & 60631,4 & 14,7 & 1963 & 17133,5 & 0,9 & 29427,7 & 2,2 \\
\hline 1945 & 56031,3 & 14,1 & 1964 & 14878,5 & 0,8 & 26011,2 & 1,8 \\
\hline 1946 & 48778,0 & 11,5 & 1965 & 13384,1 & 0,7 & 85029,0 & 5,8 \\
\hline 1947 & 45637,3 & 12,1 & 1966 & 13760,3 & 0,6 & 24074,9 & 1,5 \\
\hline 1948 & 46015,1 & 11,0 & 1967 & 14612,3 & 0,5 & 24769,5 & 1,3 \\
\hline 1949 & 44954,6 & 10,7 & 1968 & 31961,2 & 1,1 & 89426,3 & 4,0 \\
\hline 1950 & 38043,7 & 8,9 & 1969 & 86655,2 & 2,4 & 163909,6 & 6,6 \\
\hline 1951 & 29019,2 & 8,5 & 1970 & 110937,4 & 2,8 & 219795,1 & 7,3 \\
\hline 1952 & 36630,9 & 7,8 & 1971 & 112185,4 & 3,0 & 161597,6 & 5,7 \\
\hline 1953 & 34437,0 & 6,5 & 1972 & 186057,0 & 4,3 & 199505,9 & 5,8 \\
\hline 1954 & 35633,2 & 6,6 & & & & & \\
\hline
\end{tabular}

Fuente: ídem figura 1.

$1185-3$.

28 Fue adquirido en 1913 para diversas obras. AMSCT. Presupuesto ordinario de 1913, caja

29 Fue solicitado al Banco de Crédito Local en 1925 y concedido en 1928. Se destinó a la mejora y ampliación del alcantarillado, distribución de aguas, obras en el puente del barranco de Santos, obras en la calle de San Sebastián, plano de la ciudad, pavimentación y a otras obras. AMSCT. Expediente reconversión de la deuda por el Banco de Crédito Local, caja 194.

30 AMSCT. Expediente reconversión de la deuda por el Banco de Crédito Local, caja 194. 
Del mismo modo, un expediente de 1940, referido a la sección 2. ${ }^{a}$ del Negociado de Hacienda, muestra un arreglo de la deuda con el Banco de Crédito Local $^{31}$. Consistió en una reestructuración del empréstito de 10 millones, así como la emisión de un paquete de obligaciones para cubrir la amortización de la Deuda interior a través de 12600 obligaciones de 500 pesetas cada una; el tipo de interés legal quedó reducido del $5 \%$ al $4 \%$ a partir de $1940^{32}$.

El Ayuntamiento también emitió en 1942 un empréstito de 13300000 pesetas «al 5\% de interés anual, amortizable a cincuenta años, con destino a la conversión, unificación y ampliación de las Deudas Municipales y plan extraordinario de obras $»^{33}$. De esta cantidad, siete millones debían destinarse a obras de urbanización y construcción de centros escolares, para lo que la Delegación de Hacienda aprobó los correspondientes presupuestos extraordinarios. El resto se empleó en regularizar la deuda, costear obras de primer establecimiento y reducir el paro obrero.

Ahora bien, a pesar de que desde 1939 se produjo una notable reducción de las operaciones de crédito municipal como consecuencia de la reestructuración de la deuda, desde 1941 hasta el final del período el descenso en este capítulo fue considerable por la propia política impuesta por la Dictadura en materia de hacienda municipal, fundamentada en el objetivo de no asumir nuevas deudas.

Entre 1959 y 1972 y debido a la estructura económica de los presupuestos, para calcular el total de deuda se ha utilizado el capítulo cuarto, de Deuda. También se han tomado, dentro del capítulo sexto, de Gastos extraordinarios y de capital, los artículos relacionados con la actividad financiera del Ayuntamiento, como el tercero, de Amortización de deuda emitida; el cuarto, de Amortización de anticipos y préstamos de entes público; y el quinto, de Amortización de anticipos y préstamos de entidades de créditos. Y como consecuencia de las lagunas existentes en las fuentes documentales utilizadas, las liquidaciones se han complementado con los presupuestos ordinarios, en un intento por computar la totalidad de la deuda de la Corporación.

Así, se observa que las cantidades liquidadas y las presupuestadas manifestaron una tendencia decreciente durante la primera década de los sesenta. Su proporción sobre el gasto liquidado se situó en torno al 1\% desde 1963 hasta 1967 (cf. tabla 2), en consonancia con la tendencia observada en la etapa anterior de reducción del volumen de deuda impuesta por la política económica del régimen en materia de endeudamiento local. Esta dinámica cambió luego de 1968; asistimos

${ }^{31}$ La política de arreglo de la deuda vino marcada por el poder central y tuvo su base en las reformas hacendísticas promovidas por el ministro José Larraz. La ley de 6 octubre de 1939 y la Ley de 9 de marzo de 1940 establecieron las pautas que debían seguirse para la recuperación de las cuentas públicas tras los efectos de la Guerra Civil. Así, se procedió a la conversión de las deudas amortizables a largo plazo que tuvieran tipos de interés iguales o superiores al $4,5 \%$, por una deuda al $4 \%$ de interés, libre de impuestos y demás cargas. Además, se suspendió la amortización de la deuda hasta el ejercicio presupuestario de 1946. Véase Comín, Francisco y López, Santiago: «Las dos Haciendas Públicas y la financiación de la Guerra Civil (1936-1939)», Hacienda Pública Española. Monográfico, 2002, pp. 155-156.

32 AMSCT. Expediente reconversión de la deuda por el Banco de Crédito Local, caja 194.

33 AMSCT. Borradores Empréstito Municipal 1942, caja 4.3.1.1. 
ahora a un incremento de las cantidades liquidadas, de modo que se pasó de casi 32000 millones de pesetas a algo más de 186000 millones en 1972. Y en lo que respecta a la proporción de la deuda, se confirma la misma conducta, pues a partir de la década de los setenta se alcanzaron los mayores niveles y de forma especial en 1972 , con un 4,2\%.

Entre las operaciones de crédito municipales reseñables de esta etapa se encuentra la concertación de un crédito con el Banco de Crédito Local en 1958, amparado en la Orden de 13 de junio de 1957, por la que se dictaron normas para el aumento de los sueldos mínimos de los funcionarios. De hecho, un expediente

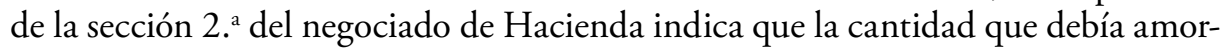
tizarse ascendía a 1804831,65 pesetas $^{34}$, a un tipo de interés del $4 \%$ y una tasa de comisiones del 0,4\%. Posteriormente, en julio de 1959 el Banco de Crédito Local remitió un telegrama al alcalde indicando que se incrementaba el tipo de interés del $4 \%$ al $4,5 \%$.

La Corporación continuaba pagando otras obligaciones, como los créditos de los años cincuenta contratados con el Banco de Crédito Local y con el Instituto Nacional de la Vivienda (INV) para la construcción de viviendas sociales en la barriada de la Victoria, en la del General García Escámez y en la de Cuesta de Piedra, con diferentes cantidades y plazos de amortización ${ }^{35}$. Finalmente, para la construcción de una piscina deportiva, cuyas obras fueron subastadas en $1962^{36}$, se suscribió un préstamo con la Delegación Nacional de Educación Física y Deporte, que ascendía a 1,5 millones de pesetas, con un tipo de interés del 5\%.

\subsection{LAS INVERSIONES}

Durante el primer franquismo las inversiones en servicios básicos marcaron una trayectoria descendente hasta 1942. Posteriormente se incrementaron hasta el final del período, al pasar de 153380 millones de pesetas a 279506 millones en 1958, lo que representa una tasa de crecimiento anual acumulativo del $4,1 \%$ (cf. figura 4 ).

Las inversiones en el capítulo de beneficencia se redujeron, pues el Ayuntamiento dejó de tener control sobre la gestión de los hospitales del municipio, que pasaron a otras entidades: el provincial (Hospital de los Desamparados ${ }^{37}$ ), perte-

34 AMSCT. Expediente de solicitudes de préstamos al Banco de Crédito Local de España con motivo del aumento de sueldo a los funcionarios. Año 1957, caja 4.3.1.12. Teniendo en cuenta el carácter histórico de algunas de las operaciones de crédito que se mantenían en vigor durante el período estudiado, se ha decidido mantener su valor en pesetas corrientes, pues no desvirtúa las cifras en el contexto analizado y, por el contrario, puede resultar bastante relevante.

35 AMSCT. Expediente de solicitudes de préstamos (57), caja 4.3.1.12.

${ }^{36}$ La subasta se hizo por 5121686,15 pesetas para un plazo de ejecución de 15 meses. Véase Resolución del Ayuntamiento de Santa Cruz de Tenerife de 4 de julio de 1962.

${ }^{37}$ Fundado en 30 de abril de 1745 por los canónigos Rodrigo e Ignacio Logman. Véase OJEDA: ob. cit. No obstante, su capacidad era insuficiente para cubrir la demanda existente. Desde 1922 se habían solicitado reformas para el recinto, pero solo se aplicaron mejoras parciales hasta que en 
Figura 4. Evolución de las inversiones y \% sobre el total de gastos liquidados, 1936-1958 (en miles de pesetas constantes de 2000).

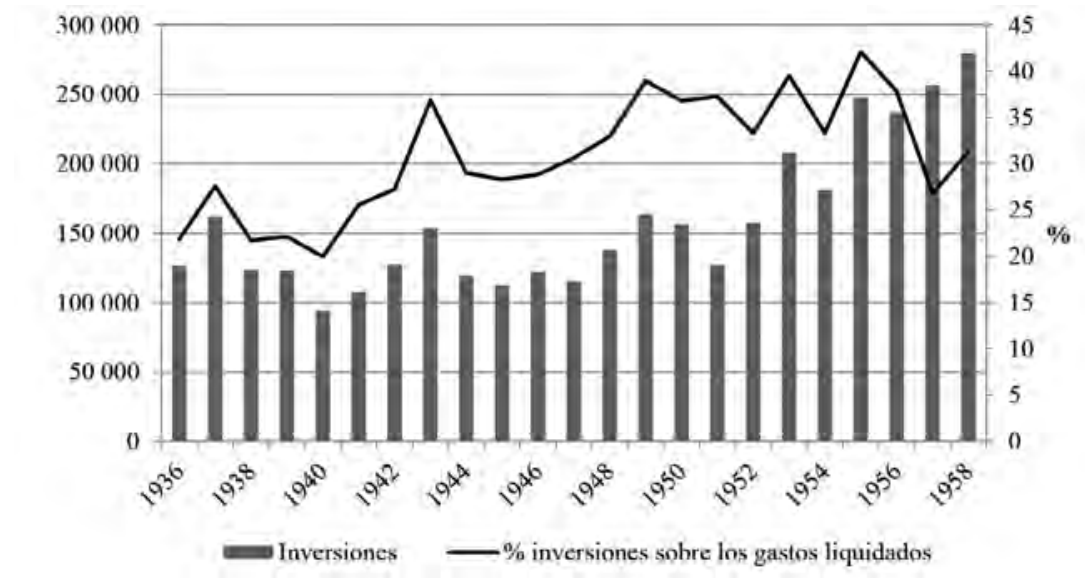

Fuente: ídem figura 1.

neciente al Cabildo Insular; el Hospital de niños ${ }^{38}$, de propiedad particular, y el Hospital militar ${ }^{39}$, propiedad del Estado.

Por su parte, las inversiones en asistencia social se incrementaron, adquiriendo un papel destacado el fomento de casas baratas ${ }^{40}$. Cabe, no obstante, observar que los escasos proyectos diseñados durante el primer franquismo tuvieron su base en los de la II República, si bien ahora se adaptaron a la filosofía franquista de proporcionar protección a las clases trabajadoras. De hecho, los organismos locales, amparándose en la Ley del Suelo de 1956, facilitaron esta tarea municipal al permitir que los municipios pudieran recurrir a empresas privadas para efectuar la construcción de dichas viviendas, lo que quedó doblemente reforzado mediante la Ley de Arrendamientos Urbanos. Además, fue en estos años cuando el municipio de Santa Cruz aprobó la elaboración del Plan Parcial del Sector Tercero-Segundo ${ }^{41}$, orientado precisamente a esta política inversora.

Por otro lado, también los ayuntamientos debían asegurar a sus empleados, mejorar sus pensiones mediante subidas de las cuotas patronales y fomentar y auxiliar

1971 se construyó un hospital general en la zona de Taco y La Cuesta. Véase Cioranescu, Alejandro: Historia de Santa Cruz de Tenerife, 1803-1977, t. 3 y 4, Santa Cruz de Tenerife, 1998, pp. 168-169.

${ }^{38}$ Inaugurado el 26 de mayo de 1901. Véase Campos, Ricardo, Montiel, Luis y Huertas, Rafael: Medicina, ideología e historia en España (siglos XVI-XXI), Madrid, 2007, p. 257.

39 Su construcción data de 1878-91. Véase Tous, Juan: Santa Cruz de Tenerife a través de la Cartografía (1588-1899), Santa Cruz de Tenerife, 1994, p. 68.

40 Estatuto Municipal de 1924, art. 211, p. 1245.

${ }^{41}$ García, Luz Marina: Propiedad del suelo en Santa Cruz de Tenerife, Santa Cruz de Tenerife, 1989, pp. 87-92. 
los montepíos ${ }^{42}$. La Ley de Régimen Local contempló, asimismo, la asistencia pública de los ancianos, que pasó a depender de la red asistencial de las dDiputaciones provinciales y de otras instituciones, aunque el nivel de prestaciones sociales fue paupérrimo ${ }^{43}$. No obstante, si bien la Dictadura promovió de forma sustitutiva los seguros laborales de tipo mixto gestionados por el Instituto Nacional de Previsión (INP), sus resultados fueron ineficaces, ante la inexistencia de una Seguridad Social moderna $^{44}$.

Los ayuntamientos también estaban obligados a dotar de una adecuada infraestructura educativa en lo que se refiere a la primera enseñanza ${ }^{45}$; $\sin$ embargo, la asignación económica de este capítulo fue siempre escasa. Así, en 1936 la inversión por habitante en instrucción pública fue de 227 pesetas, pero sus niveles mínimos se situaron en los años de mayor dureza de la autarquía -en 1947-, con tan solo 157 pesetas y una tasa de crecimiento anual acumulativo del -3,3\%. Posteriormente, esta trayectoria se invirtió al retornar a los valores cercanos al inicio del período (cf. tabla 3).

\begin{tabular}{|c|c|c|c|c|c|c|c|}
\hline \multicolumn{8}{|c|}{$\begin{array}{l}\text { TABLA 3. INVERSIÓN EN EDUCACIÓN POR HABITANTE } \\
\text { (EN PESETAS CONSTANTES DE 2000), 1936-1958 }\end{array}$} \\
\hline AÑos & INVERSIÓN & AÑos & INVERSIÓN & AÑos & INVERSIÓN & Años & INVERSIÓN \\
\hline 1936 & 227,4 & 1942 & 245,6 & 1948 & 186,9 & 1954 & 330,5 \\
\hline 1937 & & 1943 & 203,6 & 1949 & 230,0 & 1955 & 324,6 \\
\hline 1938 & & 1944 & 200,9 & 1950 & 243,2 & 1956 & 275,6 \\
\hline 1939 & & 1945 & 196,2 & 1951 & 215,1 & 1957 & 221,2 \\
\hline 1940 & 208,1 & 1946 & 186,9 & 1952 & 231,6 & 1958 & 246,8 \\
\hline 1941 & 237,0 & 1947 & 157,4 & 1953 & 267,8 & & \\
\hline
\end{tabular}

Fuente: ídem figura 1.

42 Estatuto Municipal de 1924, art. 212 y 213, p. 1245.

43 Campos, Begoña: «La construcción de una política social de vejez en España: del franquismo a la normalización democrática», Reis. Monográfico sobre sociología de la vejez, 73 (1996), pp. 243-244. En la obra se hace referencia a que García Padilla indica que las pensiones de vejez oscilaban entre las 250 y las 400 pesetas mensuales, dependiendo de si tenían derecho a la pensión complementaria de las mutualidades o montepíos.

${ }_{44} \mathrm{Al}$ parecer «la eficiencia hubiese sido mayor con una coordinación de los distintos seguros sociales, que es lo que se hacía en Europa bajo el influjo del Informe Beveridge (1942)». Véase Comín, Francisco y Díaz, Daniel: «Sector público administrativo y estado del bienestar», en Carreras, Albert y TAFunell, Xavier (coords.): Estadisticas históricas de España: siglos XIX-XX, Bilbao, pp. 892-893.

45 También, centros de enseñanza profesional, técnica o artística e instituciones culturales. Se podían concertar préstamos para este fin con el INP y sus cajas o entidades similares, que debían ceñirse al artículo 158 del Estatuto. Véase Estatuto Municipal de 1924, art. 214 y 215, p. 1245. 
Todo lo dicho revela la escasa atención dispensada por la Dictadura a la educación, en contraste con lo ocurrido durante el período anterior ${ }^{46}$. Los niveles de escolarización quedaron congelados al estallar la Guerra Civil, y el repunte de la ratio de niños escolarizados en los años cincuenta (66\%) obedeció a la reducción de la población en edad escolar por efecto de la emigración a Venezuela. Una situación que promovió la educación en centros privados, gestionados en su mayoría por órdenes religiosas. Esta realidad también se contrasta al medir la tasa de analfabetismo, que en Santa Cruz en 1950 era del 32,7\%, superior a la tasa de la provincia (27,9\%), frente al $14,2 \%$ que representaba la media nacional ${ }^{47}$.

Finalmente, las obras públicas fueron uno de los capítulos más beneficiados por la inversión realizada por el municipio. Un ámbito en el que cabe reseñar la utilización de mano de obra reclusa para su ejecución (en Tenerife, Gran Canaria y Fuertedesgracia, como denominaron a la isla de Fuerteventura). Aludimos al 91 batallón de soldados prisioneros republicanos (1500 reclusos, mayoritariamente cántabros $)^{48}$. De hecho, en Tenerife se habilitaron los almacenes de Fyffes ${ }^{49}$, que estuvieron operativos para concentrar este batallón hasta $1950^{50}$. Asimismo, el Ayuntamiento, con el apoyo del Mando Económico ${ }^{51}$, impulsó la construcción de barriadas obreras, como el barrio García Escámez, entre 1944-1945; se trataba de

46 Desde comienzos de los años veinte hasta el fin de la Segunda República, la financiación estatal de la enseñanza primaria comenzó a adquirir un protagonismo creciente, por efecto de la recuperación económica después de la Gran Guerra. De hecho, se produjo un incremento de las matrículas en la enseńanza secundaria, que pasaron de 616 en 1915 a 2384 en 1933. Véase Macías, Antonio Manuel: Voz «Educación», en Macías, Antonio Manuel (ed.): Gran Enciclopedia Canaria, Santa Cruz de Tenerife, t. 6, 1998, pp. 1351-1353.

47 Cioranescu, ob. cit., t. 4, pp. 329-331; Bergasa, Óscar y González, Antonio: Desarrollo y subdesarrollo de la economía canaria, Las Palmas de Gran Canaria, 1995, pp. 79-81.

${ }_{48}$ Recogido en la documentación de la Jefatura de Campos de Concentración y Batallones Disciplinarios del archivo militar de Ávila. Véase Pérez, José Manuel: «La transmisión de la Memoria Histórica: una propuesta didáctica», Cuadernos del Ateneo, Ejemplar dedicado a: La Memoria Histórica, 23 (2007), pp. 18-19.

${ }_{49}$ Se trataba de una firma inglesa exportadora de frutas que colaboró activamente con el régimen. Véase OJEDA: ob. cit. Las compañías consignatarias extranjeras, destacando Fyffes, ElderDempster, Wolfson y Yeoward, fueron protagonistas de la reconversión agraria de finales del siglo XIX. Arrendaban tierras a la clase terrateniente o compraban parcelas para destinarlas a los nuevos cultivos. Véase Carnero, Fernando y Nuez, Juan Sebastián: Empresa agraria y sector financiero en Canarias, c. 1852-1936, Santa Cruz de Tenerife, 2004.

50 Documental Palabras de Piel Canarias Condenados del Batallón 91: https://www.youtube. com/watch?v=P-7qn7c-W-4.

${ }^{51}$ El Mando Económico de Canarias (1941-1946) fue creado por Decreto de Presidencia del 5 de agosto de 1941. Junto con la Comandancia Militar, ostentaron un elevado nivel de poder e intervencionismo económico, al sumarse su actuación a la de los tradicionales entes (cabildos y mancomunidades). Impuso su estrategia intervencionista administrando la economía a golpe de decreto; priorizó el autoabastecimiento, lo que se tradujo en un impulso del sector agrario y en un estancamiento de las actividades vinculadas a los servicios. Véase Rodríguez, José Ångel (coord.): La Autarquia en Canarias, Santa Cruz de Tenerife, 2009, p. 22. 
viviendas protegidas para mutilados, viudas de guerra y excombatientes, a los que se añadieron ciudadanos con turno de libre elección.

La expansión de la función urbano-portuaria del municipio determinó que el esfuerzo inversor adquiriera una nueva dimensión en la década de 1960. Para valorarlo, hemos utilizado el capítulo sexto de gastos, Extraordinarios y de capital, que distinguía las inversiones productoras de ingresos y las no productoras de ingresos, y que hacen referencia a aquellas que estaban orientadas a la creación y dotación de servicios tendentes a mejorar las condiciones de vida, independientemente de que generasen o no ingresos complementarios. Ahora bien, debido a las lagunas existentes sobre las cantidades liquidadas, se ha recurrido a las cifras de los presupuestos ordinarios del Ayuntamiento sobre esta temática. Así, la proporción de ambas categorías de inversiones sobre el total de gastos presupuestados aumentó a partir de 1962, situándose entre el $15 \%$ y el $26 \%$, aproximadamente (cf. tabla 4 ).

\begin{tabular}{|c|c|c|c|c|}
\hline \multicolumn{5}{|c|}{$\begin{array}{l}\text { TABLA 4. INVERSIONES PRODUCTORAS Y NO PRODUCTORAS } \\
\text { DE INGRESOS (EN MILES DE PESETAS CONSTANTES DE 2000), 1959-1972 }\end{array}$} \\
\hline \multirow[b]{2}{*}{ AÑos } & \multicolumn{4}{|c|}{ CANTIDADES PRESUPUESTADAS } \\
\hline & $\begin{array}{l}\text { INVERSIONES NO } \\
\text { PRODUCTORAS DE INGRESOS }\end{array}$ & $\begin{array}{l}\text { INVERSIONES PRODUCTORAS } \\
\text { DE INGRESOS }\end{array}$ & TотAL & $\begin{array}{l}\% \text { SOBRE GASTOS } \\
\text { PRESUPUESTADOS }\end{array}$ \\
\hline 1959 & 7443,9 & 0,0 & 7443,9 & 0,8 \\
\hline 1960 & 0,0 & 0,0 & 0,0 & \\
\hline 1961 & 0,0 & 0,0 & 0,0 & \\
\hline 1962 & 273271,7 & 0,0 & 273271,7 & 23,2 \\
\hline 1963 & 363292,2 & 0,0 & 363292,2 & 26,8 \\
\hline 1964 & 373677,8 & 0,0 & 373677,8 & 25,9 \\
\hline 1965 & 359193,2 & 0,0 & 359193,2 & 24,5 \\
\hline 1966 & 348450,5 & 0,0 & 348450,5 & 21,2 \\
\hline 1967 & 255798,0 & 0,0 & 255798,0 & 13,0 \\
\hline 1968 & 404127,5 & 0,0 & 404127,5 & 18,3 \\
\hline 1969 & 495920,8 & 58068,5 & 553989,3 & 22,1 \\
\hline 1970 & 461235,3 & 638,4 & 461873,7 & 15,3 \\
\hline 1971 & n.d. (1) & n.d. & n.d & \\
\hline 1972 & n.d. & n.d. & n.d & \\
\hline
\end{tabular}

Nota: (1) n.d. = no hay datos.

Fuente: ídem figura 1.

Entre las inversiones de esta etapa, cabe destacar el impacto que tuvieron en Canarias los Planes de Desarrollo. Con arreglo a estos planes, se realizó un proyecto para la ejecución de obras durante 1965 y 1970, que debían integrarse en el 
Plan General de Urbanización ${ }^{52}$, y por una cuantía de 838094031,37 pesetas. El proyecto requería una inversión estimada en torno a 713357000 pesetas y en un plazo de cinco años, por lo que debía ponerse en marcha un plan de expropiación y venta de parte del patrimonio inmobiliario municipal. Asimismo, la Carta Económica Municipal debía aportar 60 millones al proyecto.

En la misma línea, la Dirección General de Puertos y Señales Marítimas autorizó al Ayuntamiento a la construcción de una playa artificial en el barrio de San Andrés ${ }^{53}$ : la playa de Las Teresitas. Se construiría un dique exterior para la contención del oleaje y otro dique interior sumergido para la contención de las arenas. El proyecto de obras ascendió a 135364266,20 pesetas, siendo aprobado en junio de $1965^{54}$. Para ello se contó con una subvención del Cabildo Insular de Tenerife de 50 millones de pesetas, una aportación municipal de 36 millones procedentes de la Carta Económica Municipal y 50 millones del producto de la enajenación de solares cedidos por los propietarios. Además, en 1972 se aprobó el Proyecto de Contrato de Préstamo ${ }^{55}$ entre el Ayuntamiento y el Banco de Crédito Local, por un importe de 50 millones. Las condiciones del contrato quedaron fijadas en un tipo de interés del $6 \%$ anual, con un plazo de diecinueve años.

\section{CONCLUSIONES}

La hacienda de Santa Cruz de Tenerife durante el primer franquismo se vio afectada por una política económica autárquica y un marco legislativo que empobrecieron a la población. Su situación presupuestaria mejoró con el Decreto refundido de 1955, que provocó un incremento de los ingresos, aunque como contraprestación la nueva estructura impositiva incrementó la presión fiscal y los ingresos liquidados por habitante.

Durante el segundo franquismo, la hacienda santacrucera se vio influenciada por la puesta en marcha del Plan de Estabilización de 1959 y por los sucesivos Planes de Desarrollo. Fue cuando se inició un proceso de cambio para adecuar la legislación fiscal a las nuevas exigencias de inversión, con el fin de afrontar las costas del desarrollismo. De hecho, fue en esta etapa cuando proliferó una legislación en materia de haciendas locales que tuvo como objetivo dotar de autosuficiencia financiera a los ayuntamientos.

En definitiva, los ingresos de la hacienda de Santa Cruz se redujeron de manera sustancial durante los años de la autarquía y, por consiguiente, el gasto municipal sufrió igual merma, fundamentalmente aquellos capítulos que afectaban

52 AMSCT. Expediente de solicitudes de préstamos, caja 4.3.1.12. de 1966.

53 Resolución de la Dirección General de Puertos y Señales Marítimas de 9 de noviembre

${ }^{54}$ AMSCT. Expediente presupuesto extraordinario ejecución playa de Las Teresitas, 1966, caja 4.3.1.93.

55 Cf. supra. 
de manera directa al bienestar de los ciudadanos. La descapitalización, la falta de inversión en bienes preferentes y el empobrecimiento municipal fueron los aspectos más visibles de la política económica del régimen. De hecho, su modelo de fiscalidad municipal tuvo como fundamento una conducta involucionista al basarse en el restablecimiento de viejas figuras asociadas a arbitrios y recargos sobre el consumo. Todo ello provocó que los niveles de deterioro socioeconómico persistieran hasta, al menos, el primer lustro de los años cincuenta para comenzar a cambiar tímidamente a finales de esta década.

El Plan de Estabilización marcó un punto de inflexión, al coincidir además con un período de expansión urbana. La nueva dinámica socioeconómica de Santa Cruz y, por supuesto, de la economía insular y regional favoreció una mejora de la renta de la población y de las propias inversiones municipales. Y en este cambio tuvo, también, su impronta el nuevo modelo de fiscalidad, así como una nueva fuente de ingresos: la Carta Económica Municipal. No obstante, todo ello no fue óbice para que, en aras de continuar con la promoción del desarrollo urbanístico de la capital, el endeudamiento tendiese a consolidarse en las cuentas del municipio.

ReCiBIDO: 07-02-2018, ACEPTADO: 10-04-2018

\section{FUENTES}

Archivo Municipal de Santa Cruz de Tenerife:

Presupuestos ordinarios de Santa Cruz de Tenerife (1936-1972).

Presupuestos liquidados de Santa Cruz de Tenerife (1936-1962 y 1972)

Expediente reconversión de la deuda por el Banco de Crédito Local.

Expediente borradores Empréstito Municipal de 1942.

Expediente de solicitudes de préstamos (57).

Expediente de solicitudes de préstamos al Banco de Crédito Local de España con motivo del aumento de sueldo a los funcionarios. Año 1957.

Expediente presupuesto extraordinario ejecución playa de Las Teresitas.

Anuarios del Mercado Español, Banesto (varios años).

Anuarios Estadísticos de España, Instituto Nacional de Estadística (varios años).

Libro de Actas del Cabildo Insular de Tenerife (varios años). 